Gynäkologe 2003 ·36:281 DOl 10.1007/s00129-003-1355-0 Online publiziert: 26. März 2003 (c) Springer-Verlag 2003

P. Hillemanns ${ }^{1} \cdot K_{\text {. Friese }}{ }^{2} \cdot$ H. Hepp ${ }^{1}$

${ }^{1}$ Klinik und Poliklinik für Frauenheilkunde und Geburtshilfe-Großhadern,

Klinikum der Universität München

${ }^{2}$ Klinik und Poliklinik für Frauenheilkunde und Geburtshilfe-Innenstadt, Klinikum der Universität München

\title{
HPV und Zervixkarzinom
}

tin belastenden Kontrolluntersuchungen $z u$ vermeiden. Im Rahmen einer großen amerikanischen Studie (ALTS) werden die differenzialdiagnostischen Testverfahren wie Kolposkopie, HPV-Test oder wiederholte zytologische Kontrollen (konventionelle oder Dünnschichtzytologie) miteinander verglichen.

\section{( ) Steht das Ende des Zervixkarzinoms bevor?}

Ein allgemein bekanntes Problem der Krebsfrüherkennung ist das in den deutschsprachigen Ländern praktizierte opportunistische Screening, das im Vergleich zu organisierten Screeningprogrammen in England oder einigen skandinavischen Ländern leider $<50 \%$ der Frauen regelmäßig nutzen. Eine Verbesserung der Teilnahmequote könnte durch die HPVSelbstuntersuchung erzielt werden, die erheblich weniger Aufwand für die Frau bedeutet. Eine Zusammenfassung der bisher publizierten Studien soll darüber Aufschluss geben, ob der HPV-Abstrich für diese Indikation geeignet ist.

Die im Editorial des New England Journal of Medicine kürzlich aufgeworfene Frage: "The beginning of the end of cervical cancer?" ist derzeit eines der spannendsten Kapitel der Onkologie überhaupt. Die ersten positiven Ergebnisse der präventiven HPV-Impfung wurden als "proof of concept" bewertet. Ob eine Impfung in den nächsten Jahren Realität wird und jetzt schon unsere Screeningstrategie in Frage stellt, diskutiert T. Grubert in seinem Beitrag.

In den molekularbiologischen Labors werden neue viel versprechende Biomarker für eine verbesserte Detektion und Diffe- renzialdiagnose der CIN evaluiert. Interessante immunhistologische, -zytologische und biochemische Nachweisverfahren stehen offensichtlich kurz vor ihrem Einsatz in klinischen und epidemiologischen Studien.

Von der AGO derzeit bereits empfohlen ist der Einsatz des HPV-Tests in der Nachsorge bei Patientinnen mit Konisation. C. Dannecker et al. vergleichen die Wertigkeit eines HPV-Tests mit der konventionellen Zytologie.

In Zeiten knapper Ressourcen im Gesundheitswesen reicht es nicht aus, wenn neue diagnostische Verfahren eine verbesserte Testgenauigkeit haben. Deshalb wird vor einer möglichen Einführung des HPVTests eine akzeptable Kosten-Nutzen-Relation gefordert.

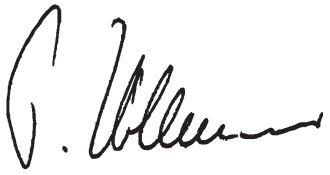

Priv.-Doz.Dr. P. Hillemanns

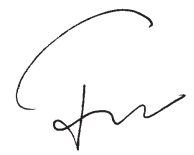

Prof. Dr. K. Friese

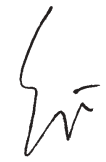

Prof.Dr. H. Hepp

\section{Korrespondierender Autor} Priv.-Doz. Dr. P. Hillemanns

Klinik und Poliklinik für Frauenheilkunde und Geburtshilfe-Großhadern, Klinikum der Universität, 81377 München E-Mail: Peter.Hillemanns@med.uni-muenchen.de zur Entscheidungshilfe bzw. Triage geeignet ist, um die zahlreichen, für die Patien- 